\title{
Sonne schützt vor Asthma
}

\section{Kinder sollten direkte Sonnenexpo- sition möglichst meiden, wird oft geraten. Aber zumindest ein paar Minuten Sonne täglich sollten sein, um den Vitamin-D-Bedarf zu decken. Ausreichend hohe Vitamin-D-Spiegel scheinen nämlich auch vor Asthma zu schützen.}

— Dies lässt sich zumindest aus epidemiologischen Untersuchungen von Wissenschaftlern der Universität Valencia (Spanien) schließen. Sie konnten zeigen, dass in Spanien das Vorkommen des chronischen Asthmas unterschiedlich verteilt ist: Im sonnenärmeren Norden Spaniens litten mehr Kinder an chronischem Asth$\mathrm{ma}$ als im sonnenreicheren Süden.

Mehr als 45000 Kinder und Teenager aus neun spanischen Städten wurden untersucht. Dabei wurde das Verhältnis der Asthmaprävalenz bei sechs- bis sieben- und 13-14-jährigen Schulkindern

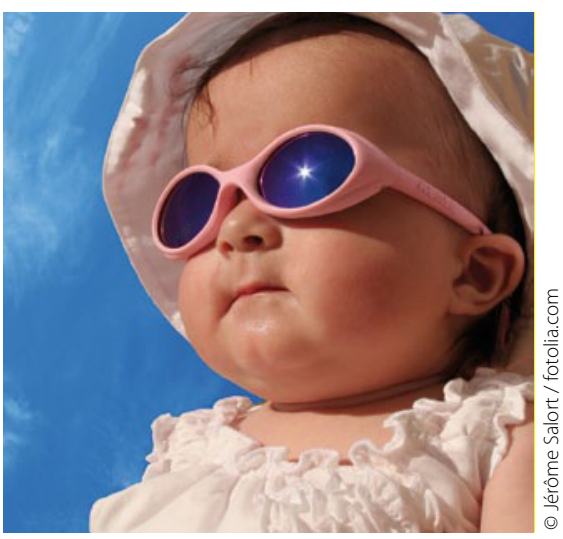

Sonnenbad für gesunde Atemwege?

zur mittleren Sonnenjahreseinstrahlung (MASH) bestimmt. Hauptbefund war: Mit einem Anstieg der MASH um $100 \mathrm{~h}$ fiel bei den jüngeren Schulkindern die Asthmaprävalenz um 0,6\% (95\%-KI: $0,4-0,8 \%$ ) und bei den älteren um $1,1 \%$ (95\%-KI: 0,8-1,3\%). In den nördlich ge- legenen Städten konnte das chronische Asthma häufiger als in den südlicheren registriert werden. Im Norden waren auch die Vitamin-D-Spiegel niedriger.

\section{Kommentar}

Tägliche Sonnenexposition ist wichtig für die Neubildung von Vitamin D. Vitamin D schützt vor Asthma, Tbc und anderen Krankheiten. Für nördliche Länder, die weniger Sonne als die Mittelmeerländer haben, wird geraten, täglich 20 bis 30 Minuten in der Sonne zu verbringen. Wegen der Hautkrebsgefahr sollte dabei die kritische Zeit zwischen mittags und vier Uhr nachmittags allerdings gemieden werden.

K. MALBERG =

\footnotetext{
- A. Arnedo-Pena et al.

Sunny hours and variations in the prevalence of asthma in schoolchildren according to the Int. Study of Asthma and Allergies (ISAAC) Phase III in Spain. Int. J. Biometrology 2011; 55 : 423-34; doi:10.1007/s00484-010-0353-x
} 